\title{
Diagnostic Value of Histological Analysis of Punch Biopsies in Suspected Cutaneous Buruli Ulcer: A Study on 32 Cases of Confirmed Buruli Ulcer in Cameroon
}

\author{
Yasmine Lucile Ibrahim ${ }^{a} \quad$ Isabelle Masouyé ${ }^{b} \quad$ Elizabeth Tschanz ${ }^{c}$ \\ Paul Atangana $^{d}$ Jean-François Etard ${ }^{f}$ Micaela Serafini $^{\mathrm{e}}$ \\ Yolanda K. Muellerg Laurence Toutous Trellu ${ }^{b}$ \\ aDivision of Clinical Pathology, Geneva University Hospitals, Geneva, Switzerland; \\ ${ }^{\mathrm{b}}$ Division of Dermatology, Geneva University Hospitals, Geneva, Switzerland; ' $V$ Viollier \\ Laboratory, Geneva, Switzerland; ${ }^{\mathrm{d}}$ Centre Pasteur Cameroon, Yaounde, Cameroon; \\ ${ }^{\mathrm{e}}$ Médecins Sans Frontières, Geneva, Switzerland; ${ }^{\mathrm{f}}$ Epicentre, Paris, France \& Institut de \\ Recherche pour le Développement (IRD) UMI 233-INSERM U 1175, Montpellier University, \\ Montpellier, France; 9 Institut Universitaire de Médecine de Famille (IUMF), Department of \\ Ambulatory Care and Community Medicine, University of Lausanne, Lausanne, Switzerland
}

Keywords

Prospective study · Buruli ulcer · Histology

\section{Abstract}

Background: Buruli ulcer (BU) is a cutaneous infectious disease caused by Mycobacterium ulcerans. In this prospective study, we aim to clarify the main histopathological features of cutaneous BU based on 4-mm skin punch biopsies and to evaluate the diagnostic value of this method. Methods: Between 2011 and 2013, a prospective study was conducted in Cameroon. Dry swabs from ulcerative lesions and fine-needle aspirates of nonulcerative lesions were examined for Ziehl-Neelsen (ZN) staining, followed by PCR targeting IS2404 and culture. Two 4-mm punch biopsies were performed in the center and in the periphery of each lesion. $\boldsymbol{R e}$ sults: The 364 patients included in the study had 422 lesions (381 were ulcerative and 357 lesions were biopsied). Among the 99 ulcerated lesions with a final diagnosis of $\mathrm{BU}$, histological features for BU were fulfilled in 32 lesions. 32/32 showed subcutaneous necrosis with a neutrophilic inflammatory infiltrate. 26/32 presented alcohol-resistant bacilli confirmed by ZN stain on histology. Conclusion: Punch biopsies help in establishing the correct diagnosis of BU and also in the differential diagnosis of chronic ulcers. The main histological feature for BU is diffuse coagulative necrosis of subcutaneous tissue, with acid-fast bacilli detected by ZN stain. 


\section{Introduction}

Mycobacterium ulcerans infection known as Buruli ulcer (BU) is a neglected tropical disease of the skin. It is prevalent in scattered foci around the world, predominantly in riverine areas with a humid, hot climate. It is an important health problem that occurs in about 33 (mostly tropical) countries, with the greatest frequency reported in Africa, particularly in West African countries. Outside Africa, cases have also been described in Japan, Mexico, South America, Papua New Guinea, and Australia [1]. In Cameroon, M. ulcerans infection has been reported in several provinces. The district of Akonolinga was identified as a health district with a high prevalence of BU, in 2007, the overall prevalence being $0.47 \%$ [2]. The mode of transmission remains poorly understood; both transmission by insects or direct inoculation by a contaminated environment has been suggested. Lesions are most commonly found on exposed parts of the body, particularly on the limbs. Recommended treatment consists of antibiotic therapy and surgical debridement, followed by skin grafting if necessary. Natural evolution of the disease may lead to spontaneous healing but, in the absence of early detection and appropriate treatment, the disease can extend leaving functional incapacities as some patients can develop osteomyelitis and articular lesions. It is known that M. ulcerans produces a toxin, mycolactone, which induces necrosis and ulceration by its cytotoxic and immunosuppressive properties [3].

BU diagnosis remains a challenge in resource-limited countries. Laboratory confirmation of suspected cases prevents misclassification, ensures adequate treatment, and provides reliable incidence and prevalence data that in turn might support the development of new disease strategies [4]. Confirmation of the clinical diagnosis becomes increasingly important to minimize erroneous antibiotic treatment and it is recommended to be used to complement clinical diagnosis [5]. Previously available data on diagnostic tests applied to different specimens suggest a wide range of sensitivities [6]. The Ziehl-Neelsen (ZN) stain detecting alcoholresistant bacilli in a swab taken from below the undermined edge of the ulcer base shows a low sensitivity rate of $40 \%$. In nonulcerative lesions, ZN stain on fine-needle aspirates can also be used to detect acid-fast bacilli (AFB) [4, 7]. Culture of $M$. ulcerans reveals a low sensitivity rate of $35-50 \%$ with many false-negative cases, similar to that of direct examination of ZN staining for AFB in smears. Microbial culture is not widely available and takes up to 8 weeks for a result $[4,7]$. PCR has become a fast and reliable method for the diagnosis of M. ulcerans infection in humans, a test whose results can be obtained within 2 days. The PCR for the IS2404 is specific to M. ulcerans DNA and is a highly sensitive method to detect $M$. ulcerans in human tissue [4, 6, 7]. A cohort study, conducted in Ghana between 2006 and 2008, assessed the sensitivity of different laboratory diagnostic tests. Across all subgroups, PCR assessment of 3-mm punch biopsy tissue specimens proved to be the best diagnostic tool for nonulcerated lesions with a sensitivity rate of $85 \%$, and PCR assessment of swab samples was also the best diagnostic tool for ulcerated lesions [6]. This method tends to be implemented in poor rural areas from where transportation is required towards a reference laboratory that can provide strict quality control and a well-trained personnel [3]. Histology mainly performed on excised lesions is more sensitive than culture and ZN stain on smears (63-90\%), but surgical excision is often not required when patients are treated with antibiotherapy $[4,8,9]$. Histological description of BU has mostly been based on case reports [10, $11]$, and only one study, published in 2003, offers a systematic description of the infected tissue on excisional skin biopsy [8]. In this paper, we aim to clarify the main histopathological features of cutaneous BU on 4-mm punch biopsies and to determine the diagnostic value of this method. 

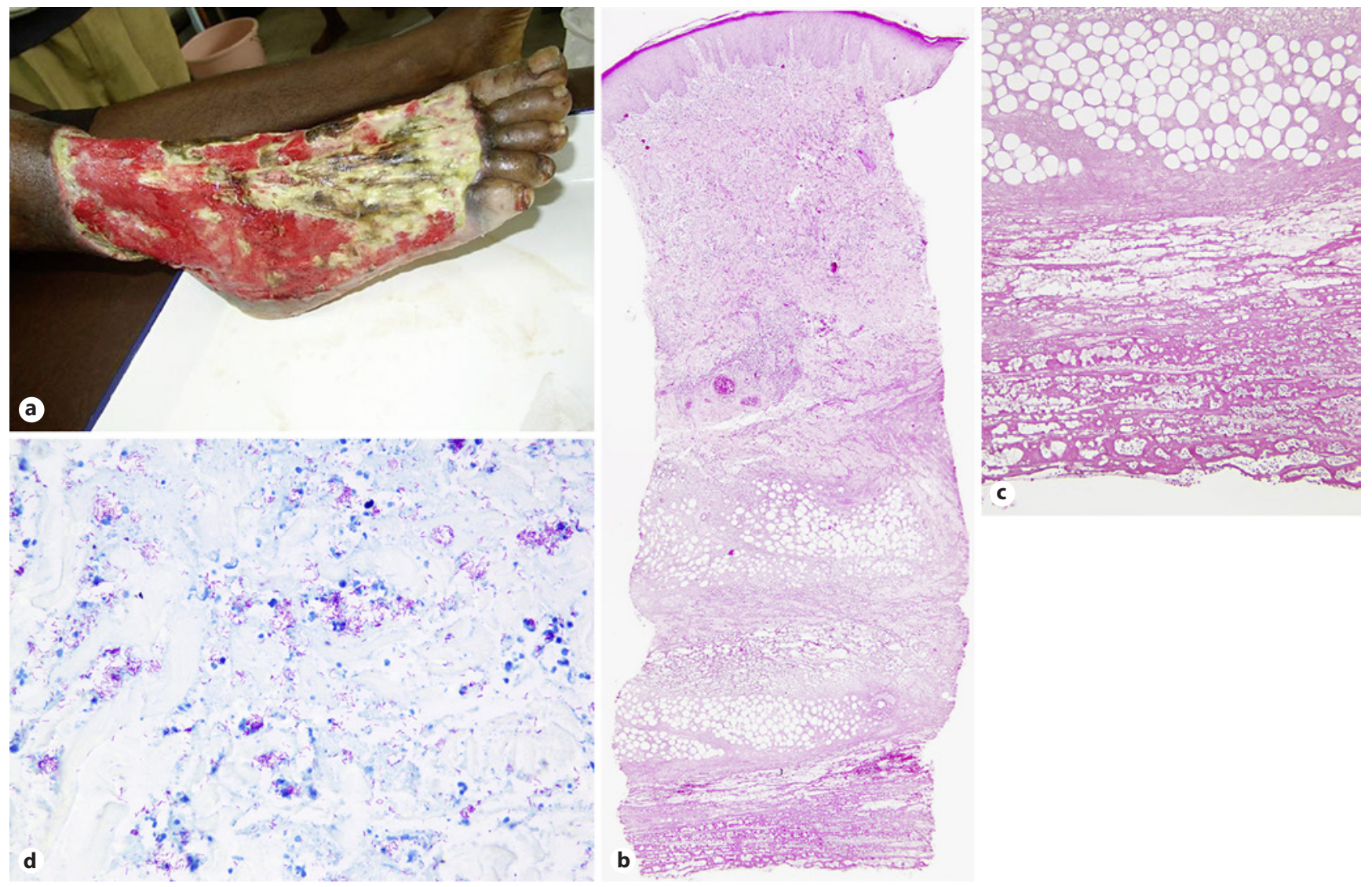

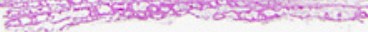

Fig. 1. a Clinical photography of a BU of the dorsum of the foot. $\mathbf{b}$ Edge of a BU at low magnification $(\times 20)$, showing a coagulative dermal and subcutaneous necrosis (hematoxylin/eosin stain). c High magnification $(\times 200)$, showing subcutaneous necrosis and acute neutrophilic inflammatory infiltrate (hematoxylin/eosin stain). d Alcohol-resistant bacilli confirmed by ZN stain (original magnification, $\times 400$ ).

\section{Materials and Methods}

Between October 2011 and December 2013, a prospective cohort study was conducted in the Akonolinga Health District, central Cameroon [12]. The funder, Médecins Sans Frontières, had a role in the study design and data collection. All individuals presenting at the Akonolinga District Hospital with a suspected cutaneous new BU lesion (defined as either a nodule, plaque, localized swelling and/or an ulcer in an individual residing or having spent at least 1 night in a known endemic area for $M$. ulcerans) were included consecutively. A dermatologist from Yaounde, Central Hospital, visited the project every 2 weeks and saw a subset of the patients. Photographs were taken of all suspected lesions. Two dermatologists reviewed the photographs of all cases independently; the first dermatologist was from Yaounde and the second was an expert in tropical skin diseases in the University Hospital of Geneva in Switzerland (Fig. 1a). Dry swabs from ulcerative lesions and fine-needle aspirates of nonulcerative lesions were examined using ZN staining in the Akonolinga Hospital laboratory the day they were collected. Another set of samples was sent weekly to the reference laboratory in Yaounde (Centre Pasteur Cameroon, CPC), where, after pooling of the samples for each lesion, ZN direct examination was repeated, followed by PCR targeting IS2404 and culture. The CPC is part of a BU external quality 
control program run by the Institute of Tropical Medicine in Antwerp, Belgium. Two 4-mm punch skin biopsies were performed by trained clinicians. On ulcerative lesions, a biopsy was taken at the edge and in the center of the lesion; for nonulcerated lesions, they were taken in the middle of the nodule (although biopsy was optional for these cases). After taking the biopsies, samples were immediately fixed and stored in $4 \%$ formaldehyde before being sent to the CPC where specimens were routinely processed and embedded in paraffin blocks. The slides were prepared and were systematically stained with hematoxylin/eosin and ZN. Additional stainings (Giemsa, silver staining) were performed in cases of other clinical suspicion (leishmaniasis, mycotic infection). A first reading of the slides was done in the CPC by a local histopathologist. The slides and the paraffin blocks were then sent to the Pathology Department of the University Hospital of Geneva for a second reading by a histopathologist; if required, additional immunohistochemical analysis was performed (particularly for tumor confirmation). Every slide was assessed for the presence or absence of histopathologic features previously evaluated in definitive and suspected BU cases in past studies [8]: the presence or absence of coagulative dermal and/ or subcutaneous necrosis, panniculitis, signs of vasculopathy with vasculitis, +/- vascular parietal necrosis, +/- thrombosis, the type of inflammation (acute neutrophilic inflammation, chronic inflammation with lymphocytes +/- plasma cells or granulomatous inflammation) and the presence or absence of AFB by ZN stain. The histological case definition of "very likely BU" required the presence of diffuse coagulative necrosis of the dermis and/ or the subcutis, with a neutrophilic inflammatory infiltrate, with or without AFB detected by ZN stain on histology [8]. All other cases without a definite diagnosis were discussed in consensus meetings that took place by videoconference between Yaounde and Geneva during the course of the study, in the presence of clinicians, dermatologists and pathologists. All clinical information and laboratory results were discussed, and clinicians were asked to estimate the probability of BU on a scale from 0 to 10. Final diagnosis of BU named "consensus diagnosis" was defined as $[12,13]$ : at least two positive laboratory tests (among positive AFB detected by ZN stain in smears in Akonolinga or in CPC, positive M. ulcerans DNA detected by PCR in CPC, or positive AFB by ZN stain on histology); or being the most likely diagnosis based on both expert reviews of photographs; or BU agreed upon as the most likely diagnosis during consensus meetings.

In this study, we focused on patients with a final diagnosis of BU by consensus who also had a histological diagnosis of "very likely BU," defined as the presence of diffuse coagulative necrosis of the dermis and/or the subcutaneous tissue, with neutrophilic inflammatory infiltrate, with the presence of AFB on histological samples detected by ZN stain, or without AFB detected by histology but with at least two other positive laboratory tests.

\section{Results}

Between October 2011 and December 2013, 367 patients were included in the study out of 447 screened and 364 patients were finally analyzed ( 3 secondary exclusions due to missing clinical data), corresponding to 422 lesions of which 381 were ulcerative $(90.3 \%)$. Detailed patient flow is presented elsewhere [13]. 357 lesions were biopsied and had a pathological examination in CPC in Yaounde (350 were ulcerative and 7 were nonulcerative). Only 353 lesions were analyzed in the Pathology Department of Geneva University Hospitals, as 4 lesions could not have pathological results although there was a biopsy (either because the slides were not sent, or they were of too low quality to be analyzed or the sample was judged too superficial to be assessed). 
Table 1. Histopathologic features of 32 confirmed BU lesions

\begin{tabular}{l|l}
\hline DOI: 10.1159/000498969 & $\begin{array}{l}\text { (c) 2019 The Author(s). Published by S. Karger AG, Basel } \\
\text { www.karger.com/dpa }\end{array}$ \\
\hline
\end{tabular}

Ibrahim et al.: Histopathological Criteria of Buruli Ulcer

\begin{tabular}{lcc}
\hline Histopathologic features & $n$ & $\%$ \\
\hline Subcutaneous tissue & & \\
$\quad$ Coagulative necrosis & $32 / 32$ & 100 \\
$\quad$ Ghost cells & $24 / 32$ & 75 \\
Inflammation & & \\
$\quad$ Neutrophilic & $32 / 32$ & 100 \\
$\quad$ Lymphocytic & $10 / 32$ & 31.2 \\
$\quad$ Eosinophilic & $18 / 32$ & 56.2 \\
Granulomas & $2 / 32$ & 6.2 \\
Vasculitis & $28 / 32$ & 87.5 \\
$\quad$ Thrombosis & $12 / 32$ & 37.5 \\
$\quad$ Necrosis of vascular wall & $30 / 32$ & 93.7 \\
AFB (ZN stain) & $26 / 32$ & 81.2 \\
\hline
\end{tabular}

Table 2. Laboratory results of the 6 confirmed BU cases without alcohol-resistant bacilli by ZN stain on histology

\begin{tabular}{lllll}
\hline \multirow{2}{*}{ Case No. } & PCR & \multicolumn{2}{l}{ ZN stain on swab in } & Culture \\
\cline { 3 - 5 } & & \multicolumn{2}{l}{ Akonolinga } & CPC \\
\hline 3 & & + & + & - \\
5 & + & + & - & + \\
14 & + & + & - & - \\
19 & + & + & - & + \\
24 & + & + & + & - \\
25 & + & + & - & - \\
\hline
\end{tabular}

\section{Histological Diagnosis}

From the 353 ulcerated and nonulcerated lesions analyzed in Geneva University Hospitals, nonspecific ulcer was the most frequent histological diagnosis in more than half of the punch biopsies analyzed $(190 / 353=53.8 \%)$, followed by non-BU infectious lesions (especially bacterial skin infection, 5.7\%), "very likely BU lesions" (44/353, 12.5\%), mixed vascular/ venous ulcers (23.2\%), and neoplastic lesions (17/353, 4.8\%) including 7 squamous cell carcinomas, 7 Kaposi sarcomas, and 3 other malignant tumors (chondrosarcoma, myxoid fibrosarcoma, and leiomyosarcoma) [13].

\section{Final Consensus Diagnosis of $B U$}

After combining consensus meetings, laboratory and clinical results, a final consensus diagnosis of BU was retained in 113/422 ulcerated and nonulcerated lesions affecting 99/364 patients $(27.2 \%)$. When restricting the analysis to ulcerative lesions, final consensus diagnosis of BU was estimated in 99/381 ulcerated lesions (25.9\%) corresponding to 87/327 patients $(26.6 \%)$.

\section{Histopathologically Confirmed BU Cases}

In this study, we focused on ulcerated lesions with a final diagnosis of BU by consensus also having a histological diagnosis of "very likely BU." From the 99 ulcerated BU lesions confirmed by consensus, histological features for BU were fulfilled in 32 lesions, biopsied from 31 patients corresponding to a sensitivity rate of $32.3 \%$. Epidermal regeneration and subcutaneous necrosis (Fig. 1b) were seen in all lesions as well as acute neutrophilic inflammatory 
Table 3. Clinical features of histologically confirmed BU patients

\begin{tabular}{|c|c|c|c|c|c|c|c|}
\hline Patient & Sex & Age, years & $\begin{array}{l}\text { History of } \\
\text { trauma }\end{array}$ & HIV & Site & $\begin{array}{l}\text { Clinical } \\
\text { form }\end{array}$ & Size, $\mathrm{cm}$ \\
\hline 1 & male & 18 & + & - & foot & ulcer & $5 \times 3$ \\
\hline 2 & male & 11 & + & - & ankle & ulcer & $10 \times 7$ \\
\hline 3 & female & 10 & - & - & thorax & ulcer & $10 \times 8$ \\
\hline 4 & female & 16 & + & - & ankle & ulcer & $4 \times 2$ \\
\hline 5 & female & 16 & - & - & forearm & ulcer & $1 \times 1$ \\
\hline 6 & male & 10 & + & - & foot & ulcer & $15 \times 10$ \\
\hline 7 & male & 3 & - & - & calf & ulcer & $3 \times 2$ \\
\hline 8 & female & 3 & - & - & leg & ulcer & $1 \times 1$ \\
\hline 9 & male & 31 & - & + & leg & ulcer & $8 \times 7$ \\
\hline 10 & female & 20 & - & - & ankle & ulcer & $2 \times 2$ \\
\hline 11 & female & 9 & - & - & calf & ulcer & $2 \times 1$ \\
\hline 12 & male & 23 & + & - & hand & ulcer & $3 \times 1$ \\
\hline 13 & male & 6 & - & - & leg & ulcer & $4 \times 4$ \\
\hline 14 & male & 39 & - & - & elbow & ulcer & $2 \times 2$ \\
\hline 15 & male & 10 & - & - & calf & ulcer & $1 \times 1$ \\
\hline \multirow[t]{2}{*}{16} & female & 25 & - & + & forearm & ulcer & $7 \times 5$ \\
\hline & & & & & knee & ulcer & $2 \times 1$ \\
\hline 17 & female & 47 & - & + & buttocks & ulcer & $20 \times 10$ \\
\hline 18 & female & 73 & + & - & ankle & ulcer & $10 \times 5$ \\
\hline 19 & male & 11 & - & - & elbow & ulcer & $7 \times 6$ \\
\hline 20 & male & 11 & - & - & ankle & ulcer & $5 \times 3$ \\
\hline 21 & female & 85 & - & - & calf & ulcer & $3 \times 2$ \\
\hline 22 & male & 42 & + & + & calf & ulcer & $4 \times 4$ \\
\hline 23 & female & 56 & - & + & foot & ulcer & $30 \times 10$ \\
\hline 24 & female & 6 & - & - & calf & ulcer & $15 \times 15$ \\
\hline 25 & female & 14 & - & - & arm & ulcer & $16 \times 10$ \\
\hline 26 & male & 45 & - & + & leg & ulcer & $17 \times 12$ \\
\hline 27 & male & 32 & - & + & foot & ulcer & $2 \times 1$ \\
\hline 28 & female & 12 & + & - & ankle & ulcer & $3 \times 2$ \\
\hline 29 & female & 6 & - & - & ankle & ulcer & $4 \times 3$ \\
\hline 30 & male & 27 & - & - & leg & ulcer & $8 \times 6$ \\
\hline 31 & male & 66 & + & + & ankle & ulcer & $7 \times 7$ \\
\hline
\end{tabular}

infiltrate (32/32) (Fig. 1c). Ghost cells could be seen in 75\% of BU lesions (24/32). Most showed signs of vasculopathies $(28 / 32)$, some with thrombi $(12 / 32)$ and many with vasculitis (28/32). 30/32 showed parietal necrosis of superficial vessels, most probably secondary to the ulcerative status of the lesions. $26 / 32$ presented alcohol-resistant bacilli confirmed by ZN stain on histological examination (Fig. 1d). The results of histological analysis of the $32 \mathrm{BU}$ lesions are shown in Table 1. The 6 cases without alcohol-resistant bacilli by ZN stain had at least two positive laboratory tests for M. ulcerans: ZN stain on swabs or on fine-needle aspirates, culture or PCR results (Table 2). 15/32 showed a positive culture for M. ulcerans. AFB in swab samples were detected in 25/32 cases. M. ulcerans DNA was identified by PCR in 31/32 cases and PCR was shown to have a sensitivity rate of $94 \%$ [13]. 31 of them were localized in the upper and lower extremities, only 1 was present on the trunk. 9 patients were HIV positive. The clinical features of histologically confirmed BU patients are shown in Table 3.

Amongst the 67 remaining ulcerated lesions with a final consensus diagnosis of $\mathrm{BU}$, 10/67 did not have any histological results, and the remaining 57 lesions showed the following histological features: absence of AFB by ZN stain (57/57), presence of focal coagulative 
necrosis of dermis +/- subcutis (32/57), presence of a chronic lymphocytic inflammatory infiltrate $(25 / 57)$ or a neutrophilic inflammatory infiltrate $(10 / 57)$, with sometimes some signs of vasculopathy probably secondary to the ulcerative status $(14 / 57)$.

\section{Discussion}

Based on the systematic histological examination of the 32 definite BU lesions, the most reliable histopathological feature for the diagnosis of $\mathrm{BU}$ in ulcerated lesions by using 4-mm punch biopsies is diffuse coagulative necrosis of dermis +/- subcutaneous tissue with a neutrophilic inflammatory infiltrate and the presence of AFB detected by ZN stain $[4,13,15]$. The subcutaneous tissue necrosis found in such cases has been attributed to an extracellular toxin, a polyketide-derived macrolide, called mycolactone, that is produced by M. ulcerans. Once the organism is introduced in the subcutaneous tissue, it proliferates and elaborates this toxin that has an affinity for fat cells. It has both cytotoxic and immunosuppressive properties resulting in necrosis and providing a favorable milieu for further proliferation of the organism. As these histological features are nonspecific and may change as the lesion evolves from a nodule to an ulcer, diagnosis of BU requires correlation with clinical data and other laboratory results [8].

Confirmation of clinically suspected cases of BU is based on the presence of any two of the following laboratory findings: presence of AFB in a smear stained by the ZN technique, positive culture of $M$. ulcerans from the infected tissue, histopathological study of the excisional biopsy specimen and evidence of $M$. ulcerans DNA by the PCR technique $[5,9,14]$. Recently, researchers have devised a new rapid diagnostic test using fluorescent thin-layer chromatography to selectively detect mycolactone. This simple and rapid test was identified as a promising technique and has the potential to become an important tool for field workers involved in BU case detection and treatment at the district level. Further studies are planned to address the logistics of introducing this test to endemic countries, to confirm its sensitivity and to investigate its specificity [18].

Our study revealed that histopathology has a sensitivity rate of $32.3 \%$ in diagnosing BU in punch biopsies of ulcerated lesions. This sensitivity is rather representative of the diagnosis of BU in endemic areas in a context of a large recruitment of cases [5]. Histopathology is a useful method for monitoring unexpected response to treatment [16] and for establishing differential diagnosis [17]. In our study, histological examination of biopsy specimens was helpful in the diagnosis of other entities presenting as cutaneous ulcers, particularly malignant neoplasia (squamous cell carcinoma, Kaposi sarcoma, leiomyosarcoma, myxoid fibrosarcoma, and chondrosarcoma) [13]. Histologic analysis also helped in diagnosing non-BU infectious ulcers and mixed vascular ulcers [13]. Punch biopsies can be easily performed in endemic areas by welltrained medical teams, and histology may be used for research purposes especially in studying the pathophysiology of paradoxical reactions and understanding the absence of response to treatments [16]. The era of performance of large surgical excisions is actually almost over with the development of earlier methods of diagnosis and early efficient medical management.

Histopathological analysis has some drawbacks as it requires an experienced laboratory relatively close to the care center and it is also quite expensive to perform [3]. The diagnostic value of punch biopsies is sometimes limited, and false-negative results could happen when samples are too superficial. Moreover, marks of traditional medicine applied in wounds before sampling have interfered with the diagnosis. Also, samples may suffer from technical problems during each step of preparation up to the analysis of slides. In summary, although histopathology is not as sensitive as PCR in diagnosing BU, histopathological analysis of suspected BU lesions by using 4-mm punch biopsies in the center and in the periphery of the lesions, by adding the ZN stain, can help in providing the correct diagnosis of BU. Our study 
shows that the most reliable histopathological features for diagnosing ulcerated BU disease on punch biopsies are diffuse necrosis of the dermis +/- subcutaneous tissue with neutrophilic inflammatory infiltrate and the presence of AFB by ZN stain. However, the histological changes are not unique, and diagnosis requires correlation with the clinical picture and other laboratory techniques. Histopathology also remains one of the best tools for establishing the differential diagnosis of chronic ulcers in endemic areas.

\section{Acknowledgements}

We would like to thank the MSF and MoH staff of the Akonolinga District Hospital and the MSF team in Yaounde. We thank Prof. Laura Rubbia-Brandt, Head of Clinical Pathology Department of Geneva University Hospital, for her academic support and the material support of her department helping in performing complementary analysis in difficult cases.

\section{Statement of Ethics}

Ethical approval was given by the National Ethics Committee of Cameroon, the Central Commission on Human Subject Research Ethics of the Geneva University Hospital, and the Ethical Review Board of Médecins Sans Frontières. The Ministry of Health, in the framework of the National Buruli Control Program, as well as the health authorities of the Akonolinga District and Akonolinga Hospital administration approved the study. All patients included gave an informed written consent.

\section{Disclosure Statement}

The authors have no conflicts of interest to disclose.

\section{References}

1 WHO. Buruli ulcer disease. Mycobacterium ulcerans infection: an overview of reported cases globally. Wkly Epidemiol Rec. 2004;79:194-200.

2 Porten K, Sailor K, Comte E, Njikap A, Sobry A, Sihom F, et al. Prevalence of Buruli ulcer in Akonolinga health district, Cameroon: results of a cross sectional survey. PLoS Negl Trop Dis. 2009 Jun;3(6):e466.

3 World Health Organization. Guidance on sampling techniques for laboratory-confirmation of Mycobacterium ulcerans infection (Buruli ulcer disease). 2010. Available from: http://www.who.int/buruli/Guidance_ sampling_techniques_MU_infection.pdf.

4 Phillips R, Horsfield C, Kuijper S, Lartey A, Tetteh I, Etuaful S, et al. Sensitivity of PCR targeting the IS2404 insertion sequence of Mycobacterium ulcerans in an Assay using punch biopsy specimens for diagnosis of Buruli ulcer. J Clin Microbiol. 2005 Aug;43(8):3650-6.

5 Mensah-Quainoo E, Yeboah-Manu D, Asebi C, Patafuor F, Ofori-Adjei D, Junghanss T, et al. Diagnosis of Mycobacterium ulcerans infection (Buruli ulcer) at a treatment centre in Ghana: a retrospective analysis of laboratory results of clinically diagnosed cases. Trop Med Int Health. 2008 Feb;13(2):191-8.

6 Herbinger KH, Adjei O, Awua-Boateng NY, Nienhuis WA, Kunaa L, Siegmund V, et al. Comparative study of the sensitivity of different diagnostic methods for the laboratory diagnosis of Buruli ulcer disease. Clin Infect Dis. 2009 Apr;48(8):1055-64.

7 Portaels F, Agular J, Fissette K, Fonteyne PA, De Beenhouwer H, de Rijk P, et al. Direct detection and identification of Mycobacterium ulcerans in clinical specimens by PCR and oligonucleotide-specific capture plate hybridization. J Clin Microbiol. 1997 May;35(5):1097-100.

8 Guarner J, Bartlett J, Whitney EA, Raghunathan PL, Stienstra Y, Asamoa K, et al. Histopathologic features of Mycobacterium ulcerans infection. Emerg Infect Dis. 2003 Jun;9(6):651-6. 
9 Portaels F, Johnson P, Meyer W. Buruli ulcer: diagnosis of Mycobacterium ulcerans disease. Geneva: World Health Organisation; 2001. Available from: http://www.who.int/gtb-buruli/publications/index.html.

10 Taheri T, et al. Buruli ulcer (skin infection with M. ulcerans): a case report and review of literature. Pathol J. 2009;41:81-2.

11 Abalos FM, Aguiar J Sr, Guédénon A, Portaels F, Meyers WM. Mycobacterium ulcerans infection (Buruli ulcer): a case report of the disseminated nonulcerative form. Ann Diagn Pathol. 2000 Dec;4(6):386-90.

12 Mueller Y, Bastard KM, Nkemenang P, et al. The Buruli Score: development of a multivariable prediction model for diagnosis of Mycobacterium ulcerans infection in individuals with ulcerative skin lesions, Akonolinga, Cameroon. PLoS Negl Trop Dis. 2016 Apr 5;10(4):e0004593.

13 Toutous Trellu L, Nkemenang P, Comte E, Ehounou G, Atangana P, Mboua DJ, et al. Differential Diagnosis of Skin Ulcers in a Mycobacterium ulcerans Endemic Area: Data from a Prospective Study in Cameroon. PLoS Negl Trop Dis. 2016 Apr;10(4):e0004385.

14 van der Werf TS, Stienstra Y, Johnson RC, Phillips R, Adjei O, Fleischer B, et al. Mycobacterium ulcerans disease. Bull World Health Organ. 2005 Oct;83(10):785-91.

15 Beissner M, Herbinger KH, Bretzel G. Laboratory diagnosis of Buruli ulcer disease. Future Microbiol. 2010 Mar; 5(3):363-70.

16 Wadagni A, et al. Simple, Rapid Mycobacterian ulcerans Disease Diagnosis from Clinical Samples by Fluorescence of Mycolactone on Thin Layer Chromatography. PLoS Negl Trop Dis. 2015;9(11):e0004247.

17 Nienhuis WA, Stienstra Y, Abass KM, Tuah W, Thompson WA, Awuah PC, et al. Paradoxical responses after start of antimicrobial treatment in Mycobacterium ulcerans infection. Clin Infect Dis. 2012 Feb;54(4):519-26.

18 Guarner J. Buruli ulcer. Review of a neglected skin mycobacterial disease. J Clin Microbiol. 2018 Mar; 56(4):e01507-17. 